\title{
El Estatuto de las Cooperativas del Mercosur
}

\author{
Dante Cracogna \\ Universidad de Buenos Aires
}

Recibido: $\quad 18.05 .09$

Aceptado: 22.06.09

Sumario: 1. El Tratado de Asunción y la naturaleza jurídica del Mercosur, - 2. Presencia cooperativa y nacimiento de la RECM,-3. El Estatuto de las Cooperativas del Mercosur. 3.1. Iniciativa y desarrollo. 3.2. Principales contenidos. 3.3. Perspectivas, -4. Anexo: Texto del Estatuto de las Cooperativas del Mercosur.

Resumen: Inicialmente se trata acerca de la estructura del Mercosur destacando la naturaleza intergubernamental de sus órganos, tal como fuera establecido por el tratado fundacional y los protocolos posteriores, a diferencia de lo que sucede en la Unión Europea. A continuación se hace referencia a la presencia del movimiento cooperativo en el proceso de integración, especialmente en el Foro Consultivo Económico y Social que promovió la constitución de la Reunión Especializada de Cooperativas del Mercosur. Este órgano viene cumpliendo una importante labor en el ámbito regional, dentro de la cual resulta especialmente importante la elaboración del proyecto de Estatuto de las Cooperativas del Mercosur destinado a ser incorporado a la legislación cooperativa de los Estados Partes. La parte final está dedicada a realizar un análisis general y particular de las disposiciones que conforman el Estatuto, recientemente aprobado por el Parlamento del Mercosur con miras a facilitar la constitución de cooperativas transfronterizas en el ámbito regional.

Palabras clave: cooperativas, Mercosur, legislación.

Abstract: The article begins referring to the structure of the Mercosur pointing out the «intergovernmental» nature of its organs according to the prescriptions of the founding Asuncion Treaty and later protocols. That situation is different from what happens in the EU. Next, the article deals with the participation of the co-operative movement in the regional integration process particularly in the Economic and Social Consultative Forum which promoted the constitution of the Specialized Meeting of Co-operatives of the Mercosur. This organ has been performing an important task in the region deserving special mention the elaboration of the project of the Statute of the Mercosur Co-operatives intended to be adopted by the national Parliaments of the member States as part of their co-operative legislation. Finally, an analysis is made both of the general aspects and particular norms of the Statute which was recently passed by the Mercosur Parliament with the aim to facilitate the constitution of transnational co-operatives within the region.

Key words: co-operatives, Mercosur, legislation. 


\section{El Tratado de Asunción y la naturaleza jurídica del Mercosur}

El art. 1. ${ }^{\circ}$ del Tratado de Asunción suscripto en 1991 que decidió la constitución del Mercado Común del Sur determina, entre otros propósitos, «el compromiso de los Estados Partes de armonizar sus legislaciones en las áreas pertinentes para lograr el fortalecimiento del proceso de integración». Es obvio que la armonización legislativa constituye un requisito conducente - y hasta indispensable - para coadyuvar a la realización del objetivo de la integración regional, lo cual fue claramente advertido por los cuatro países firmantes del Tratado a pesar de las notables dificultades que era dable advertir, ya en aquel momento, para avanzar en este terreno.

No obstante dicha declaración, de acuerdo con lo dispuesto por el Protocolo de Ouro Preto —que en 1994 estableció la estructura institucional definitiva del Mercosur - los órganos con capacidad decisoria tienen «naturaleza intergubernamental» (art. 2. ${ }^{\circ}$ ); es decir no supranacional. Por lo tanto, los Estados Partes se comprometen a «adoptar las medidas necesarias para asegurar, en sus respectivos territorios, el cumplimiento de las normas emanadas de los órganos del Mercosur» (art. 38), a cuyo efecto tales normas deberán «ser incorporadas a los ordenamientos jurídicos nacionales mediante los procedimientos previstos por la legislación de cada país» (art. 42). El art. 40 del mencionado Protocolo establece el procedimiento a seguir a fin de que las normas del Mercosur adquieran vigencia simultánea en los Estados parte. ${ }^{1}$

A las limitaciones indicadas — que excluyen la posibilidad de existencia de normas supranacionales emanadas del Mercosur- debe agregarse el complejo mecanismo previsto para la adopción de las decisiones de sus órganos (incluida la aprobación de normas), a saber: «serán tomadas por consenso y con la presencia de todos los Estados Partes» (art. 37). En la práctica ello supone la necesidad de unanimidad y permite el veto por parte de un país miembro mediante la sola inasistencia de sus representantes a las reuniones en las que se tratarán tales decisiones.

Frente a lo dicho, el recordado compromiso de los Estados Partes de «armonizar sus legislaciones en las áreas pertinentes para lograr el fortalecimiento del proceso de integración», resulta difícilmente realizable.

Por otra parte, el Parlamento del Mercosur creado por el Protocolo de Montevideo de 2005 —al igual que su antecesora, la Comisión Parlamentaria Conjunta creada por el Protocolo de Ouro Preto- no es

1 Estas circunstancias explican que exista un elevado porcentaje de normas adoptadas por los órganos del MERCOSUR que no han sido «internadas» en los Estados Partes. 
órgano con capacidad decisoria. Conforme establecen los fundamentos del Protocolo Constitutivo, se reconoce «la importancia de fortalecer el ámbito institucional de cooperación interparlamentaria, para avanzar en los objetivos previstos de armonización de las legislaciones nacionales en las áreas pertinentes y agilizar la incorporación a los respectivos ordenamientos internos de la normativa del Mercosur que requiera aprobación legislativa». Sus propósitos y competencia, tal como los definen los arts. 2 y 4 del mencionado Protocolo, se limitan a reconocerle funciones de representación; defensa de la democracia; impulso del desarrollo; formación de conciencia colectiva de valores ciudadanos; promoción de la solidaridad y la cooperación regional; etc., pero no para dictar normas de armonización legislativas ni normas legales en general. Sin embargo, el Parlamento se halla facultado para «elaborar estudios y proyectos de normas nacionales, orientados a la armonización de las legislaciones nacionales en los Estados Partes, los que serán comunicados a los Parlamentos nacionales a los efectos de su eventual consideración» (art. 4, inc. 14). Este embrión de función legislativa, en cuanto habilita al Parlamento para tomar iniciativas de leyes a efectos de someterlas a los parlamentos nacionales, constituye una novedosa función susceptible de contribuir al avance de la armonización legislativa.

\section{Presencia cooperativa y nacimiento de la RECM}

El Protocolo de Ouro Preto creó, juntamente con otros, un órgano de consulta que es el único no formado por funcionarios de los gobiernos ni por parlamentarios de los Estados Partes: el Foro Consultivo Económico y Social. Se trata de un órgano de asesoramiento integrado por representantes de la sociedad civil —en igual número por cada Estado Parte- que solamente puede expedir recomendaciones dirigidas al órgano ejecutivo del Mercosur (el Grupo Mercado Común).

El movimiento cooperativo de los diferentes países, consciente de la importancia del proceso de integración regional y de la necesidad de tomar parte activa en su desarrollo, procuró de inmediato su participación en este órgano que estuvo inicialmente formado por representantes de organizaciones sindicales de trabajadores y empleadores. Desde allí las cooperativas comenzaron a tener presencia activa en la estructura de integración y empezó asimismo a ser reconocida su importancia en este terreno por los demás sectores participantes y los gobiernos.

En 1999 el Foro Consultivo Económico y Social dirigió una recomendación al Grupo Mercado Común proponiendo la creación de una 
reunión especializada en materia de cooperativas. ${ }^{2}$ El Grupo Mercado Común, conforme con las atribuciones asignadas por el Protocolo de Ouro Preto, el 10.10.01 dictó la resolución N. ${ }^{\circ}$ 35/01 en virtud de la cual crea la «Reunión Especializada de Cooperativas» (RECM) integrada por representantes gubernamentales de los cuatro Estados Partes que actúa en coordinación con las entidades nacionales representativas del sector cooperativo de cada uno de los países.

Una notable peculiaridad de la RECM consiste en que se halla integrada por funcionarios estatales de los Estados Partes - los titulares de los organismos oficiales relacionados con las cooperativas en cada uno de ellos ${ }^{3}$ - y por los representantes de las máximas organizaciones nacionales de integración del movimiento cooperativo de cada país, ${ }^{4}$ según lo dispone el art. $2 .^{\circ}$ de la resolución mencionada.

La resolución de Grupo Mercado Común asigna a la RECM la finalidad de «analizar y desarrollar proyectos en esta área, especialmente en lo referente a la armonización de aspectos legislativos; la complementación de actividades productivas y/o de servicios; la armonización de políticas públicas del Sector Cooperativo y la promoción de la libertad de circulación e instalación de las cooperativas en la región» (art. 1. ). Cabe señalar que resolución GMC N. ${ }^{\circ}$ 35/01 no necesita ser incorporada al ordenamiento jurídico de los Estados Partes por cuanto regula aspectos de organización interna y de funcionamiento del Mercosur (art. 3. ${ }^{\circ}$ ); de manera que ella es directamente operativa desde su sanción.

Desde su creación la RECM ha venido realizando una intensa labor orientada a realizar sus objetivos relacionados con la armonización legislativa y de políticas públicas en la materia, además de la promoción de la libertad de establecimiento de las cooperativas, a cuyo efecto constituyó una Comisión Jurídica especialmente encargada de estas tareas. La primera acción consistió en lograr una aproximación seria y realista al estado actual de la legislación cooperativa en la región con miras a cono-

2 Las reuniones especializadas, al igual que los subgrupos de trabajo, son órganos que el Grupo Mercado Común puede constituir para el cumplimiento de sus objetivos, según establece el art. 14, inc. V, del Protocolo de Ouro Preto.

${ }^{3}$ Instituto Nacional de Asociativismo y Economía Social (INAES) de Argentina; Departamento Nacional de Cooperativas del Ministerio de Agricultura (DENACOOP) de Brasil; Instituto Nacional de Cooperativismo (INCOOP) de Paraguay y Comisión Honoraria de Cooperativismo $(\mathrm{CHC})$ de la Oficina de Planeamiento y Presupuesto de Uruguay.

${ }^{4}$ Confederación Cooperativa de la República Argentina (COOPERAR) y Confederación Intercooperativa Agropecuaria (CONINAGRO) de Argentina; Organización de las Cooperativas Brasileñas (OCB) de Brasil; Confederación Paraguaya de Cooperativas (CONPACOOP) de Paraguay y Confederación Uruguaya de Entidades Cooperativas (CUDECOOP) de Uruguay. 
cer claramente las similitudes y asimetrías existentes entre los diferentes países. ${ }^{5}$ Asimismo se desarrollaron seminarios y reuniones técnicas para tratar la cuestión habiéndose logrado un significativo grado de avance en el conocimiento de la materia y en la apreciación de cuáles son los principales institutos acerca de los que debería versar la armonización legislativa. Paralelamente se fueron considerando otros aspectos vinculados con la situación jurídica de las cooperativas como consecuencia del análisis de las políticas públicas a ellas referidas, especialmente en cuanto concierne a su tratamiento fiscal. ${ }^{6}$

En cuanto respecta a la libertad de establecimiento, es claro que la actuación de las cooperativas más allá de las fronteras de sus países de origen resulta problemática puesto que ellas se constituyen para prestar servicios a sus asociados; por lo tanto, su actuación en otros países implica contar en ellos con asociados a quienes brindar servicios. No se trata, simplemente, de hacer negocios como ocurre con las sociedades comerciales y si bien pueden prestar servicios a no asociados, no podría esta actividad consistir en la razón de ser exclusiva de su actuación fuera del país de origen.

La Ley de Cooperativas paraguaya N. ${ }^{\circ} 348 / 94$, sancionada después de la creación del Mercosur, contiene una disposición que autoriza a operar en ese país a las cooperativas legalmente constituidas en el extranjero y que observen los principios cooperativos, sobre la base de reciprocidad con el país de origen. Asimismo admite la constitución de cooperativas binacionales o multinacionales en el marco de la integración cooperativa (art. 23).

A su vez, la Ley argentina N. 20.337 declara aplicables a las cooperativas constituidas en el extranjero las disposiciones de la Ley de Sociedades Comerciales con relación a las sociedades extranjeras pero con las modificaciones previstas por la Ley de Cooperativas en materia de autorización para funcionar y registro (art. 15). Norma similar contiene la Ley uruguaya N. ${ }^{\circ} 18.407$ (art. 17). Asimismo, en virtud del tratado celebrado entre Brasil y Argentina en 1990 pueden constituirse en ambos países

${ }^{5}$ Como resultado se publicó: Dante Cracogna (Coordinador), Régimen legal de las cooperativas en los países del MERCOSUR, Intercoop, Buenos Aires, 2003. La segunda edición de este libro, publicada en 2005, incluye también la legislación de los Estados Asociados: Bolivia, Chile, Perú y Venezuela. Actualmente (2009) se halla en curso de publicación una tercera edición actualizada. Como antecedente merece mención el Primer Congreso de Legislación Cooperativa del MERCOSUR, realizado en Buenos Aires en 1994, cuyos Anales fueron publicados bajo el título La legislación cooperativa en el MERCOSUR, Intercoop, Buenos Aires, 1994.

${ }^{6}$ Cfr. Dante Cracogna (Coordinador), Las cooperativas y los impuestos en el Mercosur, Intercoop, Buenos Aires, 2004, passim. 
empresas binacionales argentino-brasileñas sujeto a que el capital y los inversores de cada uno de ambos países alcancen las proporciones que el tratado establece. Las empresas binacionales deben revestir alguna de las formas jurídicas admitidas por la legislación del país sede, entre las cuales se cuenta la cooperativa, y sus integrantes pueden ser personas jurídicas de derecho privado de ambos países — también cooperativascon mayoría de inversores nacionales. ${ }^{7}$

Por otra parte, la Ley argentina (art. 5. ${ }^{\circ}$ ), la Ley brasileña N. ${ }^{\circ}$ 5764/71 (art. 88) y la Ley uruguaya (art. 81) permiten que las cooperativas se asocien con personas de otro carácter jurídico para la realización de actividades vinculadas con su objeto social. Dichas personas jurídicas, especialmente bajo la forma de sociedades comerciales, podrían actuar en el extranjero conforme con las disposiciones legales de cada uno de los respectivos países.

\section{El Estatuto de las Cooperativas del Mercosur}

\subsection{Iniciativa y desarrollo}

A comienzos de 2006 la RECM emitió una Comunicación (N. ${ }^{\circ}$ 02/06) acerca de las Políticas Públicas en Materia de Cooperativas en la cual expresa la necesidad de realizar esfuerzos para lograr "que cada país adopte medidas concretas que contribuyan a posibilitar y estimular la constitución de cooperativas transfronterizas y regionales y facilitar el reconocimiento legal de las cooperativas de los otros países del Mercosur».

En razón de tales fundamentos, la RECM constituyó una Comisión Técnica encargada del estudio de un estatuto legal orientado a promover el desarrollo y la integración cooperativa en el ámbito regional del Mercosur. Dicha Comisión comenzó a funcionar en octubre de 2006 contando con la participación de parlamentarios de distintos Estados Partes que integraban la Sub Comisión de Cooperativas de la ex Comisión Parlamentaria Conjunta, a la sazón todavía existente, además de expertos en legislación cooperativa de los diferentes países.

La Comisión Técnica efectuó un amplio relevamiento de las distintas posibilidades de realizar la integración cooperativa en el ámbito regional

${ }^{7}$ Cfr. Alfredo Victorino Callejo, «Alternativas para la integración de las cooperativas en el ámbito del MERCOSUR. (El Estatuto de las Empresas Binacionales Argentino-Brasileñas)», Revista de Derecho Privado y Comunitario, N. ${ }^{\circ}$ 8, Rubinzal-Culzoni, Buenos Aires, 1995, pág. 479 y ss. 
a la luz de la normativa vigente y tomó asimismo en cuenta la experiencia del Derecho Comparado, especialmente el Estatuto de la Sociedad Cooperativa Europea (Reglamento CE 1453/2003) ${ }^{8}$ que había sido sancionado pocos años antes. Sin embargo, consideró de especial importancia contemplar la posibilidad de constituir cooperativas - de primer o segundo grado- que admitan asociados domiciliados en más de un Estado Parte; es decir, cooperativas que puedan estar formadas por personas de diferentes países del Mercosur a las que presten los servicios propios de su respectivo objeto social.

Sin perjuicio de ello, la Comisión estimó que la previsión de la existencia de estas cooperativas transfronterizas no debía excluir la posibilidad de encarar otras formas jurídicas de integración que se hallen previstas en la legislación común, particularmente referidas a las sociedades comerciales, y otros tipos de alianzas estratégicas orientadas a consolidar el movimiento cooperativo en la esfera de la integración regional. En todo caso, se trata de una manifestación que se aprecia de importancia en el camino de un proceso que admite otras posibilidades que seguramente en el futuro habrán de ser también materia de regulación legislativa. Asimismo consideró que ha de tenerse presente que la armonización tributaria en el marco regional constituye un elemento relevante para viabilizar la integración económica, incluido el campo cooperativo.

Después de los estudios y consultas realizados se arribó a la conclusión de que la forma práctica para establecer el régimen de esta peculiar clase de cooperativas consistiría en incorporar dentro de las leyes de cooperativas de los Estados Partes - mediante los procedimientos legislativos propios de cada uno de ellos - un capítulo especial dedicado a aquéllas. De esa manera no se altera el régimen común de las cooperativas $y$, por otro lado, se logra uniformidad en el tratamiento de las Cooperativas del Mercosur por parte de todos los países.

Para la implementación de este proyecto legislativo resultaba decisiva la colaboración del flamante Parlamento del Mercosur que sustituyó a la Comisión Parlamentaria Conjunta. ${ }^{9}$ A tal efecto, y habiendo contado ya con el apoyo de la mencionada Subcomisión de Cooperativas - miembros de la cual participaron en la etapa de estudio y elaboración

8 Sobre el Estatuto de la Sociedad Cooperativa puede verse, entre otros: Dante Cracogna, "Una novedad comunitaria: la Sociedad Cooperativa Europea», Revista del Derecho Comercial y de las Obligaciones, N. ${ }^{\circ} 208$, Buenos Aires, 2004, pág. 983 y ss.

${ }^{9}$ La composición del Parlamento del Mercosur durante el período de transición que corre hasta fines de 2010 es similar a la de la ex Comisión Parlamentaria Conjunta por cuanto se halla formada por igual número de parlamentarios designados por cada uno de los Parlamentos nacionales de entre sus propios miembros. 
del Proyecto- resulta aplicable el art. $4 .^{\circ}$, punto 14 , del Protocolo de Montevideo constitutivo del Parlamento del Mercosur que le asigna a éste competencia para elaborar estudios y anteproyectos de normas nacionales, orientados a la armonización de las legislaciones nacionales de los Estados Partes, para ser comunicados a los Parlamentos nacionales a los efectos de su eventual consideración.

Una vez completado el procedimiento de elaboración y consulta en el ámbito de la Comisión Técnica y de la RECM, el Proyecto fue elevado al Grupo Mercado Común el cual lo trasladó al Parlamento del Mercosur que había entrado recientemente en funcionamiento. Allí comenzó su tránsito siendo inicialmente asignado a la Comisión de Trabajo, Políticas de Empleo, Seguridad Social y Economía Social, la cual le prestó unánime aprobación en marzo de 2009 mediante Acta 1109. Previamente se había realizado en Montevideo a fines de 2008 un Seminario conjunto Parlamento del Mercosur-RECM en el que fue ampliamente analizado el proyecto por parlamentarios y miembros de la RECM. Finalmente, el Parlamento del Mercosur aprobó por unanimidad el Estatuto en su sesión del 28.4.09 realizada en Asunción, convirtiendo así a esta sanción en la primera que adopta desde su constitución. Además, para asegurar la incorporación del Estatuto a las respectivas legislaciones nacionales de los Estados Partes, en la misma oportunidad el Parlamento resolvió la creación de una comisión de seguimiento integrada por parlamentarios de cada Estado Parte y representantes de la RECM.

\subsection{Principales contenidos}

a. Régimen. El Estatuto no está concebido como un cuerpo autónomo de normas destinado a tener vigencia independiente sino que se halla previsto para ser incorporado a cada una de las legislaciones nacionales sobre cooperativas de los Estados Partes; es decir a la Ley 20.337 (1973) de Argentina; a la Ley 5764 (1971) de Brasil; a la Ley 438 (1994) de Paraguay y a la Ley 18.407 (2008) de Uruguay. Por lo tanto, no conforma un conjunto de normas diferenciadas sino un grupo de disposiciones que habrá de integrarse como una suerte de capítulo especial dentro de cada ley nacional formando parte de ella.

Es por ello que el Estatuto sólo contiene un reducido número de normas - las indispensables para caracterizar a las Cooperativas del Mercosur- puesto que estas cooperativas se regirán de manera integral por las disposiciones de las respectivas leyes de cooperativas de cada Estado Parte. No se diferenciarán del resto de las cooperativas argentinas, brasileñas, paraguayas o uruguayas sino por su denominación social (en la 
que debe incorporarse la expresión "Cooperativa del Mercosur») y las normas que sus estatutos deben incluir para adecuarse a tal condición.

De tal suerte, no existirá sujeción a normativa comunitaria ni a un cuerpo diferenciado de normas sino que todo el régimen estará contenido en la misma ley nacional de cooperativas. En efecto, el Estatuto prescribe expresamente que las Cooperativas del Mercosur se regirán por las «disposiciones comunes que rigen a las cooperativas en cuanto a su constitución, registro, funcionamiento, supervisión, disolución y liquidación» ${ }^{10}$ con la única salvedad relativa a las adecuaciones «que en razón de su naturaleza resulten del presente capítulo y fueren pertinentes a su organización y funcionamiento».

El Estatuto emplea una terminología corriente procurando de esa manera un léxico estándar para los diferentes países pero no se descarta que en cada uno de los Estados Partes se efectúe una adecuación que lo homogeinice con la terminología empleada en la legislación local.

b. Naturaleza. El motivo que da razón de ser y justifica la existencia de las Cooperativas del Mercosur radica en que las cooperativas de un Estado Parte puedan brindar sus servicios a personas domiciliadas en otro u otros Estados Parte pero no solamente extendiendo sus actividades a ellos en calidad de usuarios sino incorporándolos como asociados; es decir formando parte de la cooperativa y no como terceros ajenos. Este es el quid de la cuestión: cooperativas con asociados en dos o más Estados Partes lo cual resulta de particular interés en las áreas de fronteras donde la vecindad geográfica y la comunidad de problemas aconsejen aprovechar de la mejor manera los esfuerzos para resolverlos. Pero no se limita exclusivamente a esas situaciones.

Se prevé que los asociados de las Cooperativas del Mercosur domiciliados en el Estado de su constitución deben representar más del cincuenta por ciento del total y contar asimismo con más del cincuenta por ciento del capital social suscripto. Se entiende que no resulta justificada la existencia de una cooperativa en un Estado para reunir asociados y prestar servicios mayoritariamente en otros. De allí que si una Cooperativa del Mercosur dejara de contar con los indicados porcentajes de

10 Esta disposición resulta congruente con la CIDIP II (Convención Interamericana de Derecho Internacional Privado) en materia de sociedades comerciales suscripta en Montevideo en 1979, cuyo art. 2 establece que «la existencia, capacidad, funcionamiento y disolución de las sociedades mercantiles se rigen por la ley del lugar de su constitución» entendiendo por «ley del lugar de su constitución» la del Estado «donde se cumplan los requisitos de forma y fondo requeridos para la creación de dichas sociedades». Esta Convención ha sido ratificada por los cuatro Estados Partes. 
asociados y de capital en el país de constitución durante un lapso que supere los seis meses debe comunicarlo a la autoridad encargada del registro de cooperativas y, en tal caso, perderá su condición de tal. Debe entenderse que en ese supuesto incurren en causal de disolución pues no podrían subsistir como cooperativas comunes contando con mayoría de asociados domiciliados en otro país. Producida la disolución deben liquidarse de conformidad con el régimen legal del país sede.

Por lo tanto, estas cooperativas han de tener su domicilio en un Estado Parte y se constituirán de conformidad con su respectiva legislación de cooperativas pero previendo en sus estatutos la incorporación de asociados radicados en otro u otros Estados Partes. Para el cumplimiento de su objeto social podrán contar con establecimientos o sucursales en países diferentes del de su domicilio legal de acuerdo con el régimen común. En este aspecto, las operaciones que realicen en países distintos del de su domicilio se hallarán sujetas a las disposiciones vigentes en ellos. ${ }^{11}$

c. Nacimiento. Las Cooperativas del Mercosur pueden ser constituidas como tales desde su origen. En ese supuesto no es necesario que en el acto constitutivo participen asociados de otros Estados Partes, aunque lógicamente podrían hacerlo. Lo que se exige es que el estatuto contenga las previsiones requeridas para obtener esa condición y que su denominación la exprese. En todo caso, el trámite de constitución y reconocimiento legal de la Cooperativa del Mercosur será el mismo que la legislación nacional prevea para las cooperativas comunes.

También las Cooperativas del Mercosur pueden nacer a partir de cooperativas comunes que ya existan. Este supuesto, que previsiblemente puede tener lugar en casos de cooperativas de frontera u otras que ya tengan algún vínculo con otros países, el procedimiento consistirá en realizar una reforma del estatuto que incorpore las disposiciones pertinentes y modifique la denominación social, la cual debe ser resuelta por las asamblea mediante una mayoría de dos tercios de sus asociados presentes. Una vez adoptada la decisión por la asamblea, la cooperativa debe tramitar su inscripción en el Registro de Cooperativas de la manera que se realiza con cualquier reforma estatutaria.

El Estatuto exige que la denominación social se integre con la expresión «Cooperativa del Mercosur», lo cual resulta un requisito lógico para

11 En este punto también resultan aplicables las disposiciones de la CIDIP || en cuanto prescriben que "para el ejercicio directo o indirecto de los actos comprendidos en el objeto social de las sociedades mercantiles, éstas quedarán sujetas a la ley del Estado donde los realizaren» (art. 4). 
identificar a estas cooperativas distinguiéndolas de las comunes. Como las leyes nacionales de cada país ya contienen disposiciones acerca de la denominación de las cooperativas, éstas deben compatibilizarse con la exigencia del Estatuto. Así en la Argentina, por ejemplo, la denominación social deberá incluir la expresión «Cooperativa del Mercosur Limitada» precedida o seguida del nombre de fantasía que se adopte. De todas maneras, no es necesario ningún recaudo especial para la inscripción, la cual se realizará en el Registro de Cooperativas respectivo conforme con el procedimiento corriente en cada país.

No se halla prevista la constitución de Cooperativas del Mercosur mediante el procedimiento de fusión de cooperativas existentes en más de un Estado Parte, habida cuenta de las dificultades de orden práctico y legal que ella involucra. No obstante, cabe señalar que dicho mecanismo constituye un procedimiento idóneo para realizar la integración cooperativa por lo que seguramente habrá de ser materia de regulación futura, como sucede con el Estatuto de la Sociedad Cooperativa Europea. Pero en la etapa actual del proceso de integración regional resulta prematuro avanzar en ese terreno.

Debe dejarse a salvo, sin embargo, la posibilidad de que las Cooperativas del Mercosur puedan fusionarse con otras cooperativas - tanto comunes como del Mercosur - del mismo país de su domicilio, para lo cual no existe ningún inconveniente.

d. Federaciones. En los distintos Estados Partes es corriente la existencia de lo que genéricamente se denominan «cooperativas de segundo grado» que son aquéllas constituidas por cooperativas primarias (cuyos asociados son generalmente personas naturales o físicas). Estas cooperativas suelen adoptar diferentes denominaciones según la legislación de cada país o el uso común: federaciones, uniones, asociaciones, centrales, etc.

Si bien genéricamente son "cooperativas» y en tal sentido correspondería aplicarles las mismas disposiciones que a las demás sin necesidad de normas específicas, el Estatuto prevé expresamente que ellas también pueden constituirse como Cooperativas del Mercosur incorporando en este caso a cooperativas primarias domiciliadas en otros Estados Partes. Para su constitución y funcionamiento rigen las mismas disposiciones que para las cooperativas primarias pero con las adecuaciones de cada legislación nacional para las cooperativas de segundo grado y su denominación social deberá también llevar la expresión «Cooperativa del Mercosur».

Esta posibilidad legal permite llevar a cabo emprendimientos conjuntos entre cooperativas de diferentes Estados Partes aprovechando de esa 
manera la capacidad económica, financiera o tecnológica de las cooperativas primarias.

e. Asociados. El rasgo fundamental de las Cooperativas del Mercosur consiste - como se dijo- en que cuentan con asociados domiciliados en diferentes Estados Partes. No es necesario que el estatuto de la cooperativa identifique en cuáles de ellos; es suficiente con que exprese que pueden formar parte de la cooperativa asociados domiciliados en otros Estados Partes del Mercosur. Es de suponer que en un primer momento sean de un determinado país, pero luego podrían extenderse a otros siempre que no lleguen al cincuenta por ciento del total ni alcancen ese porcentaje del capital suscripto.

Se establece que todos los asociados, cualquiera fuera su domicilio, tendrán los mismos derechos y obligaciones sociales; es decir se impone la observancia del principio igualitario entre ellos y la regla del gobierno democrático, de tal suerte que no existan diferencias con relación a los asociados del Estado del domicilio de la cooperativa. A tal efecto se prescribe que el estatuto debe prever el régimen de participación de los asociados domiciliados en otros Estados Partes en las actividades de la cooperativa, lo cual involucra tanto el uso de los servicios como la intervención en su gestión y gobierno.

Obviamente, la organización de la participación dependerá de las características de cada cooperativa; de los servicios que constituyen su objeto social; del lugar de su domicilio; de la concentración o dispersión geográfica de los asociados domiciliados en otros países; de los medios de comunicación y transporte existentes; etc. Pero el principio que sienta el Estatuto es claro en cuanto a garantizar la igualdad de trato y la participación democrática.

f. Conflictos. Finalmente, se prevé una regla específica para determinar quién habrá de resultar competente para dirimir los conflictos que pudieran plantearse entre estas cooperativas y sus asociados. El Estatuto dispone que será competente la autoridad administrativa o judicial del lugar del domicilio de la cooperativa, conforme establezca la legislación respectiva. No se hace sino mantener el régimen común al que la cooperativa se encuentra sujeta, según lo antes explicado, puesto que conforme con la misma ley que se acuerda el reconocimiento han de resolverse los conflictos que en su seno se produzcan.

Por cierto que esta disposición no excluye la aplicación de métodos alternativos para la resolución de conflictos, tales como la mediación o el arbitraje, que el estatuto pudiera tener previstos. 
g. Reconocimiento. Las cooperativas son sujetos de derecho constituidos en un Estado de conformidad con su propia legislación, cuyo reconocimiento por los demás Estados se halla sujeto a las normas del Derecho Internacional Privado conforme sean los vínculos o tratados existentes entre ellos. Esa es la situación general, común a otros sujetos de derecho. ${ }^{12}$

Sin embargo, en este caso se trata de cooperativas que tienen específicamente previsto asociar y prestar servicios a personas domiciliadas en otros países por lo que su actuación en tales países habrá de producirse de manera más o menos regular o intensa. De allí que el Estatuto ha incorporado una norma especial que, congruente con los lineamientos generales de la materia, establece pautas precisas para el reconocimiento.

Así, dispone que las Cooperativas del Mercosur serán reconocidas de pleno derecho previa acreditación de su constitución legal; es decir que solamente se les exigirá la documentación que demuestre su constitución en el país de origen de acuerdo con la ley de cooperativas respectiva, pero nada más.

Sin embargo, se establece una condición para el reconocimiento en los términos antedichos: la reciprocidad del Estado Parte en el que la cooperativa estuviera constituida. Es decir que la legislación de éste tenga previsto igual tratamiento para el reconocimiento. Esta fórmula de la reciprocidad, corriente en el Derecho Internacional Privado, se orienta a procurar que todos los Estados Partes adopten el Estatuto, incorporándolo a su respectiva legislación

\subsection{Perspectivas}

Queda ahora por delante que, en virtud de la decisión del Parlamento del Mercosur, cada uno de los parlamentos nacionales de los Estados Partes brinde consideración y apruebe el Estatuto incorporándolo de esa forma a la legislación cooperativa del respectivo país. El procedimiento debe seguir las pautas que cada parlamento nacional tenga establecidas al efecto, de tal manera que concluya, finalmente, con una modificación de la Ley de Cooperativas incorporando como un nuevo capítulo el contenido del Estatuto de las Cooperativas del Mercosur. De esa manera, una vez que lo hayan hecho los cuatro Estados Partes, la regulación de las

12 La CIDIP II contiene normas expresas sobre reconocimiento de las sociedades comerciales que podrían considerarse genéricamente aplicables a las cooperativas. 
Cooperativas del Mercosur se habrá convertido en derecho positivo nacional de cada uno de ellos en forma similar a los demás. Es previsible que este proceso demande un tiempo considerable no tanto por la materia en cuestión sino por la circunstancia de tratarse de uno de los primeros casos en que el Parlamento del Mercosur interviene de esta manera.

\section{Anexo: Texto del Estatuto de las Cooperativas del Mercosur}

El siguiente es el capítulo a incorporar en las leyes de cooperativas de los países del Mercosur:

\section{Capítulo... \\ De las Cooperativas del Mercosur}

\section{Concepto}

Art. ... Son «Cooperativas del Mercosur» las que admiten asociados domiciliados en el país y en otro u otros Estados Partes del Mercosur. Los asociados domiciliados en el país deben representar más del cincuenta por ciento del total de asociados y del capital social suscripto. Cuando dejaran de contar con ese porcentaje durante un período superior a seis meses deberán comunicarlo a la autoridad encargada del Registro de Cooperativas y perderán la condición de «Cooperativas del Mercosur».

\section{Asociados}

Art. ...Todos los asociados, independientemente de su domicilio, tendrán los mismos derechos y obligaciones societarias, debiendo el respectivo estatuto prever el régimen de participación en las actividades de la cooperativa de los domiciliados en otros países sobre la base de igualdad jurídica.

\section{Denominación. Régimen}

Art. ... La denominación social de estas cooperativas deberá integrarse con la expresión "Cooperativa del Mercosur» y quedarán sujetas a las disposiciones comunes que rigen a las cooperativas en cuanto a su constitución, registro, funcionamiento, supervisión, disolución y liquidación, con las adecuaciones que en razón de su naturaleza resulten del presente capítulo y fueren pertinentes a su organización y funcionamiento.

\section{Constitución}

Art. ... Las «Cooperativas del Mercosur» podrán ser constituidas como tales o bien surgir a partir de una cooperativa ya existente. En 
este último caso será necesaria la decisión de la asamblea adoptada por mayoría de dos tercios de los asociados presentes y deberá modificarse el estatuto.

\section{Cooperativas de segundo grado}

Art. ... En las mismas condiciones establecidas en los artículos precedentes, las cooperativas de segundo grado (federaciones, uniones o centrales) podrán constituirse como «Cooperativas del Mercosur» incorporando como asociadas a cooperativas primarias domiciliadas en otros Estados Partes.

\section{Solución de conflictos}

Art. ... Para la solución de los conflictos que se plantearan entre las "Cooperativas del Mercosur» y sus asociados será competente la autoridad administrativa y/o judicial del lugar del domicilio de la cooperativa, según corresponda.

\section{Reconocimiento}

Art. ... Las «Cooperativas de Mercosur» constituidas en otros Estados Partes serán reconocidas de pleno derecho previa acreditación de su constitución legal. Este reconocimiento estará condicionado a la reciprocidad de tratamiento por el Estado Parte donde estuviera constituida la «Cooperativa del Mercosur».

\section{Bibliografía}

AAVV. La legislación cooperativa en el Mercosur. Anales del Primer Congreso de Legislación Cooperativa del Mercosur. Buenos Aires. 1994. Intercoop. Buenos Aires. 1994.

Alegría, Héctor. «Reconocimiento, libertad de establecimiento, sociedades y Mercosur» en Revista de Derecho Privado y Comunitario, N. ${ }^{\circ}$ 5. Buenos Aires. 1994, pág. 421 y ss.

Aguinis, Ana María. Empresas e inversiones en el Mercosur. Abeledo-Perrot. Buenos Aires. 1994.

CAllejo, Alfredo Victorino. «Alternativas para la integración de las cooperativas en el ámbito del Mercosur. (El Estatuto de las Empresas Binacionales Argentino-Brasileñas)», en Revista de Derecho Privado y Comunitario, N. ${ }^{\circ} 8$. Buenos Aires. 1995, pág. 479 y ss.

Cracogna, Dante (Coordinador). Régimen legal de las cooperativas en los países del Mercosur, 2. ${ }^{\text {a }}$ ed. Intercoop. Buenos Aires, 2005.

Cracogna, Dante (Coordinador). Las cooperativas y los impuestos en el Mercosur. Intercoop. Buenos Aires. 2004. 
Dromi, Roberto; Ekmedilan, Miguel A.; Rivera, Julio C. Derecho Comunitario. Régimen del Mercosur. Ciudad Argentina. Buenos Aires. 1996.

EtchVerRy, Raúl A.; Etcheverry, Gabriela. Mercosur, negocios y empresas. Ciudad Argentina. Buenos Aires. 2001.

Gagliardo, Mariano, Sociedades en el Mercosur. Abeledo-Perrot. Buenos Aires, ...

Velazco San Pedro, Luis Antonio (Coordinador). Mercosur y la Unión Europea: dos modelos de integración económica. Lex Nova. Valladolid. 1998. 